\title{
ON THE BAKRY-EMERY CRITERION FOR LINEAR DIFFUSIONS AND WEIGHTED POROUS MEDIA EQUATIONS*
}

\author{
J. DOLBEAULT ${ }^{\dagger}$, B. NAZARET ${ }^{\ddagger}$, AND G. SAVARÉ $^{\S}$
}

\begin{abstract}
The goal of this paper is to give a non-local sufficient condition for generalized Poincaré inequalities which extends the well-known Bakry-Emery condition. Such generalized Poincaré inequalities have been introduced by W. Beckner in the Gaussian case and provide, along the Ornstein-Uhlenbeck flow, the exponential decay of some generalized entropies which interpolate between the $L^{2}$ norm and the usual entropy. Our criterion improves on results which, for instance, can be deduced from the Bakry-Emery criterion and Holley-Stroock type perturbation results. In a second step, we apply the same strategy to non-linear equations of porous media type. This provides new interpolation inequalities and decay estimates for the solutions of the evolution problem. The criterion is again a non-local condition based on the positivity of the lowest eigenvalue of a Schrödinger operator. In both cases, we relate the Fisher information with its time derivative. Since the resulting criterion is non-local, it is better adapted to potentials with, for instance, a non-quadratic growth at infinity, or to unbounded perturbations of the potential.
\end{abstract}

Key words. parabolic equations, diffusion, Ornstein-Uhlenbeck operator, porous media, Poincaré inequality, logarithmic Sobolev inequality, convex Sobolev inequality, interpolation, decay rate, entropy, free energy, Fisher information

AMS subject classifications. 35B40; 35K55; 39B62; 35J10; 35K20; 35K65

The Bakry-Emery method [9] has been extremely successful in establishing logarithmic Sobolev inequalities [31]. It is also known to apply very well to the proof of Poincaré inequalities and inequalities which interpolate between Poincaré and logarithmic Sobolev inequalities [11, 6, 2, 21], the so-called "generalized Poincaré inequalities." The first paper on such inequalities has been written by W. Beckner in [11] in the case of a Gaussian measure. In [6], the inequalities have been slightly generalized by taking into account general $x$-dependent diffusions and by considering "convex entropies" based on a convex function $\psi$ satisfying the additional admissibility condition: $\psi^{\prime \prime} \psi^{(i v)} \geq 2\left(\psi^{\prime \prime \prime}\right)^{2}$. The proof is based on the entropy-entropy production method and the Bakry-Emery condition, [9]. The result has been slightly improved in [5], thus providing "refined inequalities" in the case $\psi(s)=\left(s^{p}-1-p(s-1)\right) /(p-1)$, $p \in(1,2)$. Other considerations on "generalized Poincaré inequalities" can be found in $[2,35,21,3]$.

The Bakry-Emery condition is a sufficient, local condition, which relies on a uniform strict log-concavity of the measure. Using perturbation techniques, it is possible to relax such a strict assumption to some extent, see [32, 21]. When Poincaré and logarithmic Sobolev inequalities are known to hold simultaneously, further interpolation inequalities can also be established: see for instance $[35,15,10,3]$.

\footnotetext{
*Received: December 13, 2007; accepted: April 14, 2008. Communicated by Eric Carlen.

${ }^{\dagger}$ Ceremade (UMR CNRS no. 7534), Université Paris-Dauphine, place de Lattre de Tassigny, 75775 Paris Cédex 16, France (dolbeaul@ceremade.dauphine.fr). http://www.ceremade.dauphine.fr/ dolbeaul/

${ }^{\ddagger}$ Ceremade (UMR CNRS no. 7534), Université Paris-Dauphine, place de Lattre de Tassigny, 75775 Paris Cédex 16, France (nazaret@ceremade.dauphine.fr). http://www.ceremade.dauphine.fr/ nazaret/

$\S$ Università degli studi di Pavia, Department of Mathematics, Via Ferrata 1, 27100, Pavia, Italy (savare@imati.cnr.it).http://www.imati.cnr.it/ savare/
} 
In case of generalized Poincaré inequalities, many techniques which are available for Poincaré inequalities and spectral gap approaches can be adapted, and more flexibility is available than for logarithmic Sobolev inequalities. See [6, 35, 5, 21]. Our first main result is that the Bakry-Emery condition can be replaced by a non-local condition which amounts to assuming the positivity of the first eigenvalue of a Schrödinger operator. This criterion gives only a sufficient condition, but improves on a very popular method, as will be illustrated by two examples.

The second main result of this paper is concerned with interpolation inequalities and decay estimates for the solutions of a weighted porous media equation. In [23], del Pino and the first author proved by variational techniques that sharp constants in some Gagliardo-Nirenberg interpolation were equivalent to sharp exponential decay rates of the generalized entropy of Ralston and Newman [40, 37] along the flow associated to the porous media equation in the euclidean space. A similar result was achieved in [19] by an entropy - entropy production method, and later extended in $[17,18,20]$. See also [8], and [4] for a review. A very nice interpretation in terms of a gradient flow was done first in the limit case of the heat equation in [33], and then in the porous media case in [38]. See also [1, 25, 36, 39, 28] for further results based on Wasserstein's distance and mass transportation theory. Concerning interpolation inequalities, weights and asymptotic behavior, we also have to quote $[13,22,12]$ for some recent results.

In connection with probability theory, entropy - entropy production methods have been successfully applied in a quite general framework of Riemanian manifolds: see $[7,24]$. However, our approach is more related to a series of attempts which have been made to establish rates of decay for solutions of diffusion equations of second and higher order: see $[27,16,34]$. More specifically, in [16], the question of how generalized Poincaré inequalities can be adapted to solutions of the porous media equation on the one-dimensional periodic interval was studied by direct methods, and later extended in [26] using capacity - measure tools. The main originality of our second result is probably that it relates interpolation inequalities to a linear eigenvalue problem, and therefore provides a non-local criterion, which is again an extension of the ideas of Bakry-Emery. This criterion is the same as in the linear case.

We will not discuss here the question of the regularity of the solutions and the well-posedness of the Cauchy problem. The interested reader will find in [41] an up-todate overview of results concerning the porous media equation, and further references therein. In this paper we will only focus on the derivation of functional inequalities in connection with decay estimates for entropy and entropy production estimates of smooth positive solutions of diffusion equations. We shall assume that the solutions are smooth and behave adequately under integrations by parts. The functional inequalities then hold in the functional spaces for which all integral quantities are well defined, as can be shown by a standard density argument. Decay estimates for the solutions of the evolution problems should rather be considered as an a priori estimate which defines a special class of solutions to the Cauchy problem. Such estimates are again standard in the framework of an approximation scheme.

\section{A nonlocal criterion for the Ornstein-Uhlenbeck flow}

1.1. Notations and preliminary computations. Consider on a domain $\Omega \subset \mathbb{R}^{d}$ the measure $d \gamma=g d x$ for some measurable function $g=e^{-F}$ and define the Ornstein-Uhlenbeck operator $\Delta_{g}$ by $\Delta_{g} v:=\Delta v-\mathrm{D} F \cdot \mathrm{D} v$. The fundamental property 
of this operator is that, for any $v \in H_{0}^{1}(\Omega, d \gamma)$,

$$
\int_{\Omega}|\mathrm{D} v|^{2} d \gamma=-\int_{\Omega} v \Delta_{g} v d \gamma
$$

For simplicity, we assume that $\partial \Omega$ is of class $C^{1}$ and that $g$ is smooth enough so that the trace of $g$ is well defined on $\partial \Omega$. We shall therefore consider the measure $g d \mathcal{H}^{d-1}$ induced by $d \gamma$ on $\partial \Omega$ and assume that regularity is such that integrations by parts can be performed without further precautions. Here $H_{0}^{1}(\Omega, d \gamma)$ is the space $\{w \in$ $W_{\text {loc }}^{1,1}\left(\mathbb{R}^{d}, d x\right) \cap L^{2}(\Omega, d \gamma):\|u\|_{L^{2}(\partial \Omega, d \gamma)}=0$ and $\left.\|D u\|_{L^{2}(\Omega, d \gamma)}<\infty\right\}$. Let $v$ be a positive smooth solution of the equation

$$
v_{t}=\Delta_{g} v, \quad x \in \Omega, t \in \mathbb{R}^{+}
$$

satisfying homogeneous Neumann boundary conditions

$$
\nabla v \cdot n=0 \quad x \in \partial \Omega, t \in \mathbb{R}^{+},
$$

where $n=n(x)$ denotes the unit outgoing normal vector at $x \in \partial \Omega$. Define for $p \in(1,2]$ the functionals

$$
\begin{aligned}
\mathcal{E}_{p}(t) & :=\frac{1}{p-1} \int_{\Omega}\left[v^{p}-1-p(v-1)\right] d \gamma \\
\mathcal{I}_{p}(t) & :=\frac{4}{p} \int_{\Omega}|\mathrm{D} s|^{2} d \gamma \\
\mathcal{K}_{p}(t) & :=\int_{\Omega}\left|\Delta_{g} s\right|^{2} d \gamma+\alpha \int_{\Omega} \Delta_{g} s \frac{|\mathrm{D} s|^{2}}{s} d \gamma
\end{aligned}
$$

with $s:=v^{p / 2}$ and $\alpha:=(2-p) / p$. The limit case $p=1$ is also covered, with $v=s^{2}$,

$$
\mathcal{E}_{1}(t):=\int_{\Omega}[v \log v-(v-1)] d \gamma,
$$

and $\mathcal{I}_{1}$ and $\mathcal{K}_{1}$ as above. We shall say that $\mathcal{E}_{p}, p \in[1,2]$, is the generalized entropy, or simply the entropy, since the case $p>1$ is clearly a generalization of the case $p=1$. Written in terms of $s$, for $p>1$ the entropy is

$$
\mathcal{E}_{p}=\frac{1}{p-1} \int_{\Omega}\left[s^{2}-1-p\left(s^{2 / p}-1\right)\right] d \gamma
$$

and the evolution is governed by

$$
s_{t}=\Delta_{g} s+\alpha \frac{|\mathrm{D} s|^{2}}{s} .
$$

Using (1.1) and (1.3), a computation shows that

$$
\begin{aligned}
\frac{d}{d t} \mathcal{E}_{p}(t) & :=-\mathcal{I}_{p}(t), \\
\frac{d}{d t} \mathcal{I}_{p}(t) & :=-\frac{8}{p} \mathcal{K}_{p}(t) .
\end{aligned}
$$

The next step consists in establishing a functional inequality relating $\mathcal{I}_{p}$ and $\mathcal{K}_{p}$. Using the commutation relation

$$
\left[\mathrm{D}, \Delta_{g}\right] s=-\mathrm{D}^{2} F \mathrm{D} s
$$


and integration by parts, one gets

$$
\begin{aligned}
& \int_{\Omega}\left|\Delta_{g} s\right|^{2} d \gamma \\
& \quad=\int_{\Omega}\left|\mathrm{D}^{2} s\right|^{2} d \gamma+\int_{\Omega} \mathrm{D}^{2} F \mathrm{D} s \cdot \mathrm{D} s d \gamma-\sum_{i, j=1}^{d} \int_{\partial \Omega} \partial_{i j}^{2} s \partial_{i} s n_{j} g d \mathcal{H}^{d-1}
\end{aligned}
$$

if Neumann boundary conditions hold.

Lemma 1.1. With the above notations, if $\Omega$ is convex, then

$$
-\sum_{i, j=1}^{d} \int_{\partial \Omega} \partial_{i j}^{2} s \partial_{i} s n_{j} g d \mathcal{H}^{d-1} \geq 0 .
$$

For a proof of this result, see [29, Lemma 5.2] or [30].

Hence, if $\Omega$ is convex, we have

$$
\int_{\Omega}\left|\Delta_{g} s\right|^{2} d \gamma \geq \int_{\Omega}\left|\mathrm{D}^{2} s\right|^{2} d \gamma+\int_{\Omega} \mathrm{D}^{2} F \mathrm{D} s \cdot \mathrm{D} s d \gamma .
$$

With the notation $z:=\sqrt{s}$, we get

$$
\mathrm{D} z=\frac{\mathrm{D} s}{2 \sqrt{s}}
$$

and, using integration by parts again, since $2 \mathrm{D}^{2} z: \mathrm{D} z \otimes \mathrm{D} z=\mathrm{D}\left(|\mathrm{D} z|^{2}\right): \mathrm{D} z$,

$$
4 \int_{\Omega}|D z|^{4} d \gamma=\int_{\Omega} \Delta_{g} s|\mathrm{D} z|^{2} d \gamma+2 \int_{\Omega} \mathrm{D}^{2} s: \mathrm{D} z \otimes \mathrm{D} z d \gamma .
$$

Hence by (1.4) and (1.5) we have

$$
\begin{aligned}
\mathcal{K}_{p} & =\int_{\Omega}\left|\Delta_{g} s\right|^{2} d \gamma+4 \alpha \int_{\Omega} \Delta_{g} s|\mathrm{D} z|^{2} d \gamma \\
\geq & \int_{\Omega}\left|\mathrm{D}^{2} s\right|^{2} d \gamma+\int_{\Omega} \mathrm{D}^{2} F \mathrm{D} s \cdot \mathrm{D} s d \gamma \\
& \quad+4^{2} \alpha \int_{\Omega}|D z|^{4} d \gamma-2 \cdot 4 \alpha \int_{\Omega} \mathrm{D}^{2} s: \mathrm{D} z \otimes \mathrm{D} z d \gamma .
\end{aligned}
$$

Expanding the square, we get

$$
\begin{aligned}
& \int_{\Omega}\left|\mathrm{D}^{2} s-4 \mathrm{D} z \otimes \mathrm{D} z\right|^{2} d \gamma \\
= & \int_{\Omega}\left|\mathrm{D}^{2} s\right|^{2} d \gamma-2 \cdot 4 \int_{\Omega} \mathrm{D}^{2} s: \mathrm{D} z \otimes \mathrm{D} z d \gamma+4^{2} \int_{\Omega}|D z|^{4} d \gamma,
\end{aligned}
$$

which gives the identity

$$
\begin{aligned}
& 4^{2} \alpha \int_{\Omega}|D z|^{4} d \gamma-2 \cdot 4 \alpha \int_{\Omega} \mathrm{D}^{2} s: \mathrm{D} z \otimes \mathrm{D} z d \gamma \\
= & \alpha \int_{\Omega}\left|\mathrm{D}^{2} s-4 \mathrm{D} z \otimes \mathrm{D} z\right|^{2} d \gamma-\alpha \int_{\Omega}\left|\mathrm{D}^{2} s\right|^{2} d \gamma
\end{aligned}
$$


and hence

$$
\mathcal{K}_{p} \geq(1-\alpha) \int_{\Omega}\left|\mathrm{D}^{2} s\right|^{2} d \gamma+\int_{\Omega} \mathrm{D}^{2} F \mathrm{D} s \cdot \mathrm{D} s d \gamma
$$

For $p=1$, only the last term of the right hand side is left. For $p>1$, the coefficient $1-\alpha=2(p-1) / p$ is also positive, which allows to state a nonlocal result.

\subsection{A nonlocal result. Let}

$$
V(x):=\inf _{\xi \in S^{d-1}}\left(\mathrm{D}^{2} F(x) \xi, \xi\right)
$$

and define

$$
\lambda_{1}(p):=\inf _{w \in H^{1}(\Omega, d \gamma) \backslash\{0\}} \frac{\int_{\Omega}\left(2 \frac{p-1}{p}|\mathrm{D} w|^{2}+V|w|^{2}\right) d \gamma}{\int_{\Omega}|w|^{2} d \gamma} .
$$

Notice that the above criterion is given with respect to the measure $d \gamma$, and $H^{1}(\Omega, d \gamma)$ is defined as the space $\left\{w \in W_{\mathrm{loc}}^{1,1}\left(\mathbb{R}^{d}, d x\right) \cap L^{2}(\Omega, d \gamma):\|D u\|_{L^{2}(\Omega, d \gamma)}<\infty\right\}$. If we assume that $F \in C^{2}(\bar{\Omega})$ is such that

$$
D F \cdot n \geq 0 \quad \text { on } \quad \partial \Omega
$$

(or $\Omega=\mathbb{R}^{d}$ ), and consider

$$
u:=w e^{-F / 2},
$$

a simple integration by parts provides a sufficient condition for the positivity of $\lambda_{1}(p)$ in terms of the positivity of the ground state level of a Schrödinger equation in the usual sense:

$$
\geq 2 \frac{p-1}{p} \inf _{w \in H^{1}(\Omega, d x) \backslash\{0\}} \frac{\int_{\Omega}\left[|\mathrm{D} u|^{2}+\left(\frac{p}{2(p-1)} V+\frac{1}{4}|\mathrm{D} F|^{2}-\frac{1}{2} \Delta F\right)|u|^{2}\right] d x}{\int_{\Omega}|u|^{2} d x} .
$$

Notice that the above condition is only a sufficient condition for relating $\mathcal{I}_{p}$ and $\mathcal{K}_{p}$ through (1.6). We are indeed reducing the problem to a scalar eigenvalue problem and dropping the fact that $\mathrm{D} s \cdot n=0$ on $\partial \Omega$.

ThEOREM 1.2. Assume that $F \in C^{2}(\bar{\Omega}), e^{-F} \in L^{1}(\Omega)$, where $\Omega$ is a convex domain in $\mathbb{R}^{d}$. Consider a smooth solution of (1.1) with Neumann boundary conditions on $\partial \Omega$ and an initial datum $v_{0} \in L_{+}^{1}(\Omega)$ such that $\mathcal{E}_{p}(0)$ is finite for some $p \in[1,2]$. With the above notations, if

$$
\lambda_{1}(p)>0
$$

then the solution of (1.1) is such that, for any $t \geq 0$,

$$
\begin{aligned}
& \mathcal{I}_{p}(t) \leq \mathcal{I}_{p}(0) e^{-2 \lambda_{1}(p) t}, \\
& \mathcal{E}_{p}(t) \leq \mathcal{E}_{p}(0) e^{-2 \lambda_{1}(p) t} .
\end{aligned}
$$


Proof. The first inequality is a consequence of (1.6). We may indeed write

$$
\begin{aligned}
-\frac{p}{8} \frac{d}{d t} \mathcal{I}_{p}(t)=\mathcal{K}_{p} & \geq(1-\alpha) \int_{\Omega}\left|\mathrm{D}^{2} s\right|^{2} d \gamma+\int_{\Omega} V(x)|\mathrm{D} s|^{2} d \gamma \\
& \geq \lambda_{1}(p) \int_{\Omega}|\mathrm{D} s|^{2} d \gamma=2 \lambda_{1}(p) \frac{p}{8} \mathcal{I}_{p}(t)
\end{aligned}
$$

and use a Gronwall lemma to prove the first inequality. The second inequality then follows from

$$
\frac{d}{d t}\left[\mathcal{I}_{p}(t)-2 \lambda_{1}(p) \mathcal{E}_{p}(t)\right] \leq 0
$$

the fact that

$$
\lim _{t \rightarrow \infty} \mathcal{I}_{p}(t)=0
$$

and, as a consequence,

$$
\lim _{t \rightarrow \infty}\|v(t)-\bar{v}\|_{L^{1}(\Omega, d \gamma)}=0, \quad \text { where } \quad \bar{v}=\frac{\int_{\Omega} v_{0} d \gamma}{\int_{\Omega} d \gamma},
$$

so that

$$
\lim _{t \rightarrow \infty} \mathcal{E}_{p}(t)=0 .
$$

An integration on $(t, \infty)$ then gives

$$
\frac{d}{d t} \mathcal{E}_{p}(t)-2 \lambda_{1}(p) \mathcal{E}_{p}(t)=\mathcal{I}_{p}(t)-2 \lambda_{1}(p) \mathcal{E}_{p}(t) \geq \lim _{t \rightarrow \infty}\left(\mathcal{I}_{p}(t)-2 \lambda_{1}(p) \mathcal{E}_{p}(t)\right)=0,
$$

and one again concludes with a Gronwall lemma.

As a standard consequence, this proves a generalized Poincaré inequality. Notice that the result was already known at least in case $p=2$, see, e.g., [2, Proposition 5.5.4]. How the so-called super-integral criterion for the logarithmic Sobolev inequality, see [2, Proposition 5.5.6], is related to our approach in the limit $p \rightarrow 1$ is still an open question. The boundedness of $d \gamma$, that is $e^{-F} \in L^{1}(\Omega, d x)$, is used only to obtain (1.8). Whenever (1.8) can be established by other means, the method also adapts to unbounded measures.

Corollary 1.3. Assume that $F \in C^{2}(\bar{\Omega}), e^{-F} \in L^{1}(\Omega)$, where $\Omega$ is a convex domain in $\mathbb{R}^{d}$. If $\lambda_{1}(p)$ is positive, then

$$
\frac{1}{p-1}\left[\int_{\Omega}|u|^{2} d \gamma-\left(\int_{\Omega}|u|^{\frac{2}{p}} d \gamma\right)^{p}\right] \leq \frac{2}{\lambda_{1}(p)} \int_{\Omega}|\nabla u|^{2} d \gamma \quad \forall u \in H^{1}(\Omega, d \gamma) .
$$

Proof. As a straightforward consequence of the proof of Theorem 1.2, namely according to (1.9) taken at $t=0$, we have

$$
\mathcal{E}_{p}(t=0) \leq \frac{1}{2 \lambda_{1}(p)} \mathcal{I}_{p}(t=0)
$$


which means

$$
\frac{1}{p-1} \int_{\Omega}\left[v_{0}^{p}-1-p\left(v_{0}-1\right)\right] d \gamma \leq \frac{1}{2 \lambda_{1}(p)} \frac{4}{p} \int_{\Omega}\left|\mathrm{D} v_{0}^{p / 2}\right|^{2} d \gamma
$$

for any initial value $v_{0}$ of the Cauchy problem associated to (1.1). Let $u:=\mu v_{0}^{p / 2}$. The inequality now takes the form

$$
\frac{1}{p-1} \int_{\Omega}\left[u^{2}-\mu^{2}-p\left(u^{\frac{2}{p}} \mu^{2 \frac{p-1}{p}}-\mu^{2}\right)\right] d \gamma \leq \frac{1}{2 \lambda_{1}(p)} \frac{4}{p} \int_{\Omega}|\mathrm{D} u|^{2} d \gamma .
$$

If we observe that the left hand side, as a function of $\mu$, can be written

$$
h(\mu)+\frac{1}{p-1} \int_{\Omega}|u|^{2} d \gamma
$$

where $h(\mu):=\mu^{2}-\frac{p}{p-1}|\bar{u}|^{\frac{2}{p}} \mu^{2 \frac{p-1}{p}}$ and $\bar{u}=\left(\int_{\Omega} u^{\frac{2}{p}} d \gamma\right)^{\frac{p}{2}}$, an optimization of $h(\mu)$ in terms of $\mu$ shows that

$$
h(\mu) \geq h(\bar{u})=-\frac{1}{p-1}|\bar{u}|^{2} \quad \forall \mu>0,
$$

which completes the proof.

One can notice that under the local (uniform) strict convexity condition

$$
\inf _{\xi \in S^{d-1}}\left(\mathrm{D}^{2} F(x) \xi, \xi\right)=: \lambda_{1}^{\infty}>0
$$

one recovers the usual results corresponding to the Bakry-Emery criterion [9], since in such a case $\lambda_{1}(p) \geq \lambda_{1}^{\infty}$ for any $p \in[1,2]$. Our approach does not cover all cases. If $\Omega$ is bounded and $F$ is constant, the infimum on $H^{1}(\Omega, d \gamma) \backslash\{0\}$ in the definition of $\lambda_{1}(p)$ is zero, which is achieved by constant functions. In such a case, it is therefore crucial to take into account the condition $\mathrm{D} s \cdot n=0$ on $\partial \Omega$.

If $V$ is nonnegative, $\lambda_{1}(p) \geq(p-1) \lambda_{1}(2)$. The inequality

$$
\frac{1}{p-1}\left[\int_{\Omega}|u|^{2} d \gamma-\left(\int_{\Omega}|u|^{\frac{2}{p}} d \gamma\right)^{p}\right] \leq \frac{2}{(p-1) \lambda_{1}(2)} \int_{\Omega}|\nabla u|^{2} d \gamma \quad \forall u \in H^{1}(\Omega, d \gamma)
$$

also holds for any $p \in(1,2)$ if it holds for $p=2$, even if $V$ changes sign. Using Hölder's inequality and the fact that $d \gamma$ is a probability measure, it is indeed not difficult to see that

$$
\int_{\Omega}|u|^{2} d \gamma-\left(\int_{\Omega}|u|^{\frac{2}{p}} d \gamma\right)^{p} \leq \int_{\Omega}|u|^{2} d \gamma-\left(\int_{\Omega}|u| d \gamma\right)^{2} .
$$

All results which can be achieved for $p=2$ can therefore be extended to $p \in(1,2)$, with a constant that can be bounded by the same constant as for $p=2$, up to a factor $1 /(p-1)$. However, requiring that $\lambda_{1}(p)>0$ gives much better estimates: first of all, it allows to pass to the limit case $p=1$ in the standard case of the Bakry-Emery method, that is, when $\lambda_{1}^{\infty}>0$, with a better constant for any $p>1$. Moreover, it allows to directly deal with unbounded perturbations or limit cases, as illustrated by the two following examples. 


\subsection{Two examples.}

1) Unbounded perturbations. Let $F(x)=\frac{1}{2}|x|^{2}+\varepsilon \log |x|, \Omega=\mathbb{R}^{d}$ for some $d \geq 3$. The problem cannot be reduced to the harmonic potential case by the HolleyStroock perturbation lemma, see [32,6,14], since at the origin $x=0$ and as $|x| \rightarrow \infty$, the perturbation $\varepsilon \log |x|=F(x)-\frac{1}{2}|x|^{2}$ is clearly unbounded. Assume that $d \geq 3$. The measure $d \gamma$ is bounded for any $\varepsilon \in(0, d)$. With $\nu:=\frac{p}{2(p-1)} \geq 1$, using Hardy's inequality and the expression of the first eigenvalue for the quantum harmonic oscillator, we can write that

$$
\begin{aligned}
& \int_{\Omega}\left[|\mathrm{D} u|^{2}+\left(\nu V+\frac{1}{4}|\mathrm{D} F|^{2}-\frac{1}{2} \Delta F\right)|u|^{2}\right] d x \\
= & \left(1-\mathrm{a}^{2}\right) \underbrace{\int_{\Omega}\left[|\mathrm{D} u|^{2}-\frac{(d-2)^{2}}{4|x|^{2}}|u|^{2}\right] d x}_{\geq 0}+\underbrace{\int_{\Omega}\left[\mathrm{a}^{2}|\mathrm{D} u|^{2}+\frac{|x|^{2}}{4}|u|^{2}\right] d x}_{\geq \frac{d}{2} \mathrm{a} \int_{\Omega}|u|^{2} d x}+\left(\nu-\frac{d-\varepsilon}{2}\right) \int_{\Omega}|u|^{2} d x,
\end{aligned}
$$

with a such that $\left(1-\mathrm{a}^{2}\right) \frac{(d-2)^{2}}{4}=\frac{\varepsilon}{4}(2 \mathrm{~b}-\varepsilon)$ and $\mathrm{b}:=2 \nu+d-2$. This can be done if and only if $0 \leq 1-\mathrm{a}^{2}=\frac{\varepsilon}{(d-2)^{2}}(2 \mathrm{~b}-\varepsilon) \leq 1$, that is,

$$
\varepsilon \leq \mathrm{b}-\sqrt{\mathrm{b}^{2}-(d-2)^{2}} .
$$

The case $\varepsilon \geq \mathrm{b}+\sqrt{\mathrm{b}^{2}-(d-2)^{2}}$ has indeed to be excluded because $\mathrm{b} \geq d>\varepsilon$. For $\nu>\frac{d}{2}$, i.e., $p<\frac{d}{d-1}$, the last term of the right hand side in (1.12) is positive, which proves that $\lambda_{1}(p)>0$ under condition (1.13). As a special case, this means that an upper bound for $\varepsilon$ is of the order of $\frac{(d-2)^{2}}{4 \nu}=\frac{(d-2)^{2}}{2 p}(p-1)$ as $p \rightarrow 1$. For $\nu \leq \frac{d}{2}$, we can evaluate $\lambda_{1}(p)$ from below by $\frac{d}{2} \mathrm{a}+\nu-\frac{d-\varepsilon}{2}$, which is positive for $\varepsilon>0$, small enough.

2) Semi-classical estimates. The criterion of Theorem 1.2 amounts to looking for the ground state of

$$
-\Delta u+\left(\nu V+\frac{1}{4}|\mathrm{D} F|^{2}-\frac{1}{2} \Delta F\right) u=\nu \lambda_{1}(p) u
$$

with $\nu:=\frac{p}{2(p-1)} \geq 1$ as above, and determining $\lambda_{1}(p)$. Let $F(x)=\frac{1}{\beta}|x|^{\beta}, \beta \in(1,2)$, $\Omega=\mathbb{R}$. In such a case, the problem is reduced to

$$
-u^{\prime \prime}+\left[\left(\nu-\frac{1}{2}\right)(\beta-1)|x|^{\beta-2}+\frac{1}{4}|x|^{2(\beta-1)}\right] u=\nu \lambda_{1}(p) u .
$$

It readily follows that the criterion of Theorem 1.2 covers the range $1<\beta<2$, which is not the case of the usual Bakry-Emery criterion, and a semi-classical expansion shows that

$$
\lambda_{1}(p) \sim \kappa_{\beta}\left(\nu-\frac{1}{2}\right)^{\frac{2}{\beta}(\beta-1)} \nu^{-1}=O\left((p-1)^{\frac{2-\beta}{\beta}}\right) \quad \text { as } \quad p \rightarrow 1_{+}
$$

for some positive constant $\kappa_{\beta}$ which depends only on $\beta$. Such an estimate is definitely better than the one that can be deduced from (1.11). 
1.4. A refined result in the limit case. Consider the case for which $\lambda_{1}(p)=$ 0 , but assume that for some $\varepsilon>0$,

$$
\inf _{w \in H^{1}(\Omega, d \gamma)} \frac{\int_{\Omega}\left((1-\alpha(1+\varepsilon))|\mathrm{D} w|^{2}+V|w|^{2}\right) d \gamma}{\int_{\Omega}|w|^{2} d \gamma}=0 .
$$

Recall that

$$
\begin{aligned}
\mathcal{K}_{p} \geq \int_{\Omega}\left|\mathrm{D}^{2} s\right|^{2} d \gamma+\int_{\Omega} \mathrm{D}^{2} F \mathrm{D} s \cdot \mathrm{D} s d \gamma \\
\quad+4^{2} \alpha \int_{\Omega}|D z|^{4} d \gamma-2 \cdot 4 \alpha \int_{\Omega} \mathrm{D}^{2} s: \mathrm{D} z \otimes \mathrm{D} z d \gamma .
\end{aligned}
$$

Expanding the square, we get

$$
\begin{aligned}
& \int_{\Omega}\left|\sqrt{1+\varepsilon} \mathrm{D}^{2} s-\frac{4}{\sqrt{1+\varepsilon}} \mathrm{D} z \otimes \mathrm{D} z\right|^{2} d \gamma \\
= & (1+\varepsilon) \int_{\Omega}\left|\mathrm{D}^{2} s\right|^{2} d \gamma-2 \cdot 4 \int_{\Omega} \mathrm{D}^{2} s: \mathrm{D} z \otimes \mathrm{D} z d \gamma+\frac{4^{2}}{1+\varepsilon} \int_{\Omega}|D z|^{4} d \gamma,
\end{aligned}
$$

which gives the identity

$$
\begin{aligned}
& 4^{2} \alpha \int_{\Omega}|D z|^{4} d \gamma-2 \cdot 4 \alpha \int_{\Omega} \mathrm{D}^{2} s: \mathrm{D} z \otimes \mathrm{D} z d \gamma \\
& =\alpha \int_{\Omega}\left|\sqrt{1+\varepsilon} \mathrm{D}^{2} s-\frac{4}{\sqrt{1+\varepsilon}} \mathrm{D} z \otimes \mathrm{D} z\right|^{2} d \gamma-\alpha(1+\varepsilon) \int_{\Omega}\left|\mathrm{D}^{2} s\right|^{2} d \gamma \\
& \quad+4^{2} \alpha \frac{\varepsilon}{1+\varepsilon} \int_{\Omega}|D z|^{4} d \gamma .
\end{aligned}
$$

Summarizing, we have

$$
\mathcal{K}_{p} \geq 4^{2} \frac{\alpha \varepsilon}{1+\varepsilon} \int_{\Omega}|D z|^{4} d \gamma
$$

For $p \in(1,2)$, i.e., $\alpha \in(0,1)$, we follow the method of [5]. Assume that $d \gamma$ is a probability measure and normalize $v$ in $L^{1}(\Omega, d \gamma)$ by $\int_{\Omega} v d \gamma=1$. Then using $\int_{\Omega}|s|^{2} d \gamma=1+(p-1) \mathcal{E}_{p}$, we can interpolate $\mathcal{I}_{p}$ with a Cauchy-Schwarz inequality:

$$
\left(p \mathcal{I}_{p}\right)^{2}=4^{4}\left(\int_{\Omega} s|\mathrm{D} z|^{2} d \gamma\right)^{2} \leq 4^{4}\left(1+(p-1) \mathcal{E}_{p}\right) \int_{\Omega}|\mathrm{D} z|^{4} d \gamma
$$

which gives

$$
\frac{1+\varepsilon}{\alpha \varepsilon} \mathcal{K}_{p} \geq 4^{2} \int_{\Omega}|\mathrm{D} z|^{4} d \gamma \geq\left(\frac{p}{4}\right)^{2} \frac{\mathcal{I}_{p}^{2}}{1+(p-1) \mathcal{E}_{p}},
$$

which is the functional inequality which replaces (1.10) and gives, a consequence,

$$
-\frac{\frac{d}{d t} \mathcal{I}_{p}}{\mathcal{I}_{p}^{2}} \geq \frac{\alpha \varepsilon}{1+\varepsilon} \frac{p}{2} \frac{1}{1+(p-1) \mathcal{E}_{p}(t)} \geq \frac{\alpha \varepsilon}{1+\varepsilon} \frac{p}{2} \frac{1}{1+(p-1) \mathcal{E}_{p}(0)}=: \kappa .
$$

This proves the following result. 
THEOREM 1.4. Assume that $F \in C^{2}(\bar{\Omega}), e^{-F} \in L^{1}(\Omega)$, where $\Omega$ is a convex domain in $\mathbb{R}^{d}$, and $\int_{\Omega} d \gamma=1$ so that $d \gamma$ is a probability measure. Consider a smooth solution of (1.1) with Neumann boundary conditions on $\partial \Omega$ and an initial datum $v_{0} \in L_{+}^{1}(\Omega)$ such that $\mathcal{E}_{p}(0)$ is finite for some $p \in[1,2]$. With the above notations, if $\lambda_{1}(p)=0$, then

$$
\mathcal{I}_{p}(t) \leq \frac{\mathcal{I}_{p}(0)}{1+\kappa \mathcal{I}_{p}(0) t} \quad \forall t \geq 0 .
$$

This result improves on [5], since it holds as soon as $\lambda_{1}^{\infty}=0$. In the next section, we shall use a similar method to interpolate $\mathcal{I}_{p}$ in terms of $\mathcal{E}_{p}$ and $\mathcal{K}_{p}$.

\section{Generalized entropies and nonlinear diffusion equations}

2.1. Generalized entropies. Assume now that $v$ is a nonnegative solution of the weighted porous media equation

$$
v_{t}=\Delta_{g} v^{m} .
$$

We refer for instance to [26] or [7] for such an equation. Again assume that $d \gamma$ is a probability measure and define for any $p \in(1,2)$ the entropy

$$
\frac{1}{m+p-2} \int_{\Omega}\left[v^{m+p-1}-M^{m+p-1}-(m+p-1) M^{m+p-2}(v-M)\right] d \gamma,
$$

where $M=\int_{\Omega} v(x, t) d \gamma$ does not depend on $t>0$. The results in $[12,13]$ suggest that the method could also work even if $d \gamma$ is not bounded. Taking into account the homogeneity and up to a change of the time scale, we may assume that $M=1$ and consider

$$
\begin{aligned}
\mathcal{E}_{m, p}(t) & :=\frac{1}{m+p-2} \int_{\Omega}\left[v^{m+p-1}-1\right] d \gamma \\
\mathcal{I}_{m, p}(t) & :=c(m, p) \int_{\Omega}|\mathrm{D} s|^{2} d \gamma \\
\mathcal{K}_{m, p}(t) & :=\int_{\Omega} s^{\beta(m-1)}\left|\Delta_{g} s\right|^{2} d \gamma+\alpha \int_{\Omega} s^{\beta(m-1)} \Delta_{g} s \frac{|\mathrm{D} s|^{2}}{s} d \gamma
\end{aligned}
$$

with $v=: s^{\beta}, \beta:=(p / 2+m-1)^{-1}, \alpha:=\beta m-1=\frac{2-p}{p+2(m-1)}$ and

$$
c(m, p)=\frac{4 m(m+p-1)}{(2 m+p-2)^{2}} .
$$

Written in terms of $s$, the evolution is governed by

$$
\frac{1}{m} s_{t}=s^{\beta(m-1)}\left[\Delta_{g} s+\alpha \frac{|\mathrm{D} s|^{2}}{s}\right],
$$

since

$$
\Delta_{g} s^{q}=q s^{q-1}\left(\Delta_{g} s+(q-1) \frac{|\mathrm{D} s|^{2}}{s}\right) .
$$

Using (2.1) and (2.2), a computation shows that

$$
\begin{aligned}
\frac{d}{d t} \mathcal{E}_{m, p}(t) & :=-\mathcal{I}_{m, p}(t), \\
\frac{1}{m} \frac{d}{d t} \mathcal{I}_{m, p}(t) & :=-2 c(m, p) \mathcal{K}_{m, p}(t) .
\end{aligned}
$$


2.2. A functional inequality. The next step of the method is to establish a functional inequality which replaces (1.10). In case $m=1$, we had $\mathcal{K}_{p} \geq p \lambda_{1}(p) \mathcal{I}_{p} / 4$ if $\lambda_{1}(p)>0$ (see Section 1.2). Here we find a more complicated expression which involves $\mathcal{E}_{m, p}, \mathcal{I}_{m, p}$ and $\mathcal{K}_{m, p}$, in the spirit of the computations of Section 1.4.

Using

$$
s^{q-1} \Delta_{g} s=\frac{1}{q} \Delta_{g} s^{q}-(q-1) s^{q-2}|\mathrm{D} s|^{2}=\frac{1}{q} \Delta_{g} s^{q}-\frac{4}{q^{2}}(q-1)\left|\mathrm{D} s^{q / 2}\right|^{2}
$$

with $2(q-1)=\beta(m-1)$, we can write

$$
\begin{aligned}
q^{2} \int_{\Omega} s^{\beta(m-1)}\left|\Delta_{g} s\right|^{2} d \gamma=\int_{\Omega}\left|\Delta_{g} s^{q}\right|^{2} d \gamma & +\left(\frac{4}{q}(q-1)\right)^{2} \int_{\Omega}\left|\mathrm{D} s^{q / 2}\right|^{4} d \gamma \\
& -2 \cdot \frac{4}{q}(q-1) \int_{\Omega} \Delta_{g} s^{q}\left|\mathrm{D} s^{q / 2}\right|^{2} d \gamma
\end{aligned}
$$

Assume that $\Omega$ is convex. By (1.4), we get

$$
\begin{aligned}
& \int_{\Omega}\left|\Delta_{g} s^{q}\right|^{2} d \gamma \geq \int_{\Omega}\left|\mathrm{D}^{2} s^{q}\right|^{2} d \gamma+\int_{\Omega} \mathrm{D}^{2} F \mathrm{D} s^{q} \cdot \mathrm{D} s^{q} d \gamma \\
& q^{2} \int_{\Omega} s^{\beta(m-1)}\left|\Delta_{g} s\right|^{2} d \gamma \geq \int_{\Omega}\left|\mathrm{D}^{2} s^{q}\right|^{2} d \gamma+\int_{\Omega} \mathrm{D}^{2} F \mathrm{D} s^{q} \cdot \mathrm{D} s^{q} d \gamma \\
&+\left(\frac{4}{q}(q-1)\right)^{2} \int_{\Omega}\left|\mathrm{D} s^{q / 2}\right|^{4} d \gamma \\
&-2 \cdot \frac{4}{q}(q-1) \int_{\Omega} \Delta_{g} s^{q}\left|\mathrm{D} s^{q / 2}\right|^{2} d \gamma
\end{aligned}
$$

With the notation $z:=s^{q / 2}$, this can be rewritten as

$$
\begin{aligned}
q^{2} \int_{\Omega} s^{\beta(m-1)}\left|\Delta_{g} s\right|^{2} d \gamma \geq & \int_{\Omega}\left|\mathrm{D}^{2} s^{q}\right|^{2} d \gamma+\int_{\Omega} \mathrm{D}^{2} F \mathrm{D} s^{q} \cdot \mathrm{D} s^{q} d \gamma \\
& +\left(\frac{4}{q}(q-1)\right)^{2} \int_{\Omega}|\mathrm{D} z|^{4} d \gamma \\
& -2 \cdot \frac{4}{q}(q-1) \int_{\Omega} \Delta_{g} s^{q}|\mathrm{D} z|^{2} d \gamma
\end{aligned}
$$

On the other hand, by (2.3),

$$
\begin{aligned}
& \int_{\Omega} s^{\beta(m-1)} \Delta_{g} s \frac{|\mathrm{D} s|^{2}}{s} d \gamma \\
= & \int_{\Omega} s^{q-1} \Delta_{g} s \cdot s^{q-2}|\mathrm{D} s|^{2} d \gamma \\
= & \int_{\Omega}\left(\frac{1}{q} \Delta_{g} s^{q}-\frac{4}{q^{2}}(q-1)\left|\mathrm{D} s^{q / 2}\right|^{2}\right) \cdot \frac{4}{q^{2}}\left|\mathrm{D} s^{q / 2}\right|^{2} d \gamma \\
= & \frac{4}{q^{2}} \int_{\Omega}\left(\frac{\Delta_{g} s^{q}}{q}|\mathrm{D} z|^{2}-\frac{4}{q^{2}}(q-1)|\mathrm{D} z|^{4}\right) d \gamma,
\end{aligned}
$$

where we again used the identity $2(q-1)=\beta(m-1)$. 
Collecting the estimates (2.4) and (2.5), we get

$$
\begin{aligned}
\mathcal{K}_{m, p} \geq \frac{1}{q^{2}}[ & \left.\int_{\Omega}\left|\mathrm{D}^{2} s^{q}\right|^{2} d \gamma+\int_{\Omega} \mathrm{D}^{2} F \mathrm{D} s^{q} \cdot \mathrm{D} s^{q} d \gamma\right] \\
& +\frac{1}{q^{2}}\left(\frac{4}{q}(q-1)\right)^{2} \int_{\Omega}|\mathrm{D} z|^{4} d \gamma \\
& -\frac{2}{q^{2}} \cdot \frac{4}{q}(q-1) \int_{\Omega} \Delta_{g} s^{q}|\mathrm{D} z|^{2} d \gamma \\
& +\frac{4}{q^{2}} \alpha \int_{\Omega}\left(\frac{\Delta_{g} s^{q}}{q}|\mathrm{D} z|^{2}-\frac{4}{q^{2}}(q-1)|\mathrm{D} z|^{4}\right) d \gamma \\
\mathcal{K}_{m, p} \geq & \frac{1}{q^{2}}\left[\int_{\Omega}\left|\mathrm{D}^{2} s^{q}\right|^{2} d \gamma+\int_{\Omega} \mathrm{D}^{2} F \mathrm{D} s^{q} \cdot \mathrm{D} s^{q} d \gamma\right] \\
& +16 \frac{q-1}{q^{4}}(q-1-\alpha) \int_{\Omega}|\mathrm{D} z|^{4} d \gamma \\
& +4 \frac{\alpha-2 q+2}{q^{3}} \int_{\Omega} \Delta_{g} s^{q}|\mathrm{D} z|^{2} d \gamma
\end{aligned}
$$

By definition of $z:=s^{q / 2}$, we get

$$
\Delta_{g}\left(s^{q}\right)=2|\mathrm{D} z|^{2}+2 s^{q / 2} \Delta_{g}\left(s^{q / 2}\right),
$$

so that

$$
\int_{\Omega} \Delta_{g}\left(s^{q}\right)|\mathrm{D} z|^{2} d \gamma=2 \int_{\Omega}|D z|^{4} d \gamma+2 \int_{\Omega} s^{q / 2} \Delta_{g}\left(s^{q / 2}\right)|\mathrm{D} z|^{2} d \gamma .
$$

Using integration by parts,

$$
\begin{aligned}
& \int_{\Omega} s^{q / 2} \Delta_{g}\left(s^{q / 2}\right)|\mathrm{D} z|^{2} d \gamma \\
= & -\int_{\Omega}|D z|^{4} d \gamma-2 \int_{\Omega} s^{q / 2} \mathrm{D}^{2}\left(s^{q / 2}\right): \mathrm{D} z \otimes \mathrm{D} z d \gamma \\
= & -\int_{\Omega}|D z|^{4} d \gamma-\int_{\Omega}\left[\mathrm{D}^{2}\left(s^{q}\right)-2 \mathrm{D} z \otimes \mathrm{D} z\right]: \mathrm{D} z \otimes \mathrm{D} z d \gamma \\
= & \int_{\Omega}|D z|^{4} d \gamma-\int_{\Omega} \mathrm{D}^{2}\left(s^{q}\right): \mathrm{D} z \otimes \mathrm{D} z d \gamma
\end{aligned}
$$

since

$$
\frac{1}{2} \mathrm{D}^{2}\left(s^{q}\right)=s^{q / 2} \mathrm{D}^{2}\left(s^{q / 2}\right)+\mathrm{D} z \otimes \mathrm{D} z .
$$

Hence

$$
\int_{\Omega} \Delta_{g}\left(s^{q}\right)|\mathrm{D} z|^{2} d \gamma=4 \int_{\Omega}|D z|^{4} d \gamma-2 \int_{\Omega} \mathrm{D}^{2}\left(s^{q}\right): \mathrm{D} z \otimes \mathrm{D} z d \gamma .
$$

Summarizing, with $\mathrm{b}=\frac{8}{q^{3}}(\alpha+2-2 q)$ and $\mathrm{c}=\frac{16}{q^{4}}(q-1)(q-1-\alpha)+2 b,(2.6)$ becomes

$$
\begin{aligned}
\mathcal{K}_{m, p} \geq \frac{1}{q^{2}} & {\left[\int_{\Omega}\left|\mathrm{D}^{2} s^{q}\right|^{2} d \gamma+\int_{\Omega} \mathrm{D}^{2} F \mathrm{D} s^{q} \cdot \mathrm{D} s^{q} d \gamma\right] } \\
& +\mathrm{c} \int_{\Omega}|\mathrm{D} z|^{4} d \gamma-\mathrm{b} \int_{\Omega} \mathrm{D}^{2}\left(s^{q}\right): \mathrm{D} z \otimes \mathrm{D} z d \gamma .
\end{aligned}
$$


Let

$$
V(x):=\inf _{\xi \in S^{d-1}}\left(\mathrm{D}^{2} F(x) \xi, \xi\right)
$$

and define for any $\theta \in(0,1)$

$$
\lambda_{1}(m, \theta):=\inf _{w \in H^{1}(\Omega, d \gamma) \backslash\{0\}} \frac{\int_{\Omega}\left((1-\theta)|\mathrm{D} w|^{2}+V|w|^{2}\right) d \gamma}{\int_{\Omega}|w|^{2} d \gamma} .
$$

Assume that for some $\theta \in(0,1)$,

$$
\lambda_{1}(m, \theta)>0
$$

From (2.7), we get

$$
\begin{gathered}
\mathcal{K}_{m, p} \geq \frac{\lambda_{1}(m, \theta)}{q^{2}} \int_{\Omega}\left|\mathrm{D} s^{q}\right|^{2} d \gamma+\mathrm{a} \int_{\Omega}\left|\mathrm{D}^{2} s^{q}\right|^{2} d \gamma-\mathrm{b} \int_{\Omega} \mathrm{D}^{2}\left(s^{q}\right): \mathrm{D} z \otimes \mathrm{D} z d \gamma \\
+\mathrm{c} \int_{\Omega}|\mathrm{D} z|^{4} d \gamma
\end{gathered}
$$

where $\mathrm{a}=\mathrm{a}(\theta)=\frac{\theta}{q^{2}}$. A tedious but elementary computation shows that the condition $\mathrm{b}^{2}-4 \mathrm{a}(\theta) \mathrm{c}<0$ amounts to

$$
(p+2 m-4)^{2}+\left[5 m^{2}+2(2 p-7) m+(p-3)^{2}\right] \theta<0,
$$

which defines in terms of $(m, p)$ a bounded set $\mathrm{E}_{\theta}$ such that $\partial \mathrm{E}_{\theta}$ is an ellipse. The set $\mathrm{E}_{1}$ is centered at $(1,3 / 2)$ and contained in the rectangle $[1-\sqrt{2} / 2,1+\sqrt{2} / 2] \times[0,3]$, and

$$
\mathrm{E}_{1} \cap\{(m, p): m=1\}=\{1\} \times(1,2) .
$$

See Fig. 2.1. As a function of the parameter $\theta \in(0,1)$, the family $\mathrm{E}_{\theta}$ is decreasing and $\lim _{\theta \rightarrow 0+} \overline{\mathrm{E}}_{\theta}=\{(m=1, p=2)\}$.

Coming back to the estimate of $\mathcal{K}_{m, p}$, if $(m, p) \in \mathrm{E}_{\theta}$, then

$$
\mathcal{K}_{m, p} \geq \kappa_{1} \int_{\Omega}\left|\mathrm{D} s^{q}\right|^{2} d \gamma+\kappa_{2} \int_{\Omega}|\mathrm{D} z|^{4} d \gamma
$$

with

$$
\kappa_{1}:=\frac{\lambda_{1}(m, \theta)}{q^{2}} \quad \text { and } \quad \kappa_{2}:=c-\frac{\mathrm{b}^{2}}{4 \mathrm{a}(\theta)} .
$$

The next step is based on the interpolation of $\int_{\Omega}|\mathrm{D} s|^{2} d \gamma$ between $\int_{\Omega}\left|\mathrm{D} s^{q}\right|^{2} d \gamma$, $\int_{\Omega}|\mathrm{D} z|^{4} d \gamma=\int_{\Omega}\left|\mathrm{D} s^{q / 2}\right|^{4} d \gamma$ and $(m+p-2) \mathcal{E}_{m, p}+1=\int_{\Omega} s^{1+\frac{p}{p+2(m-1)}} d \gamma$. Written in terms of $q$, we observe that $(m+p-2) \mathcal{E}_{m, p}+1=\int_{\Omega} s^{2(2-q)} d \gamma$. By applying Young's inequality with exponents 4,2 and 4 , we get

$$
\begin{aligned}
\frac{q^{2}}{2} \int_{\Omega}|\mathrm{D} s|^{2} d \gamma=\int_{\Omega} & \sqrt{A} B^{\frac{1}{4}} s^{\frac{1}{2}(4-3 q)} \frac{\mathrm{D} s^{q}}{\sqrt{A}} \cdot \frac{\mathrm{D} z}{B^{\frac{1}{4}}} d \gamma \\
\leq & \frac{A^{2} B}{4} \int_{\Omega} s^{2(4-3 q)} d \gamma+\frac{1}{2 A} \int_{\Omega}\left|\mathrm{D} s^{q}\right|^{2} d \gamma+\frac{1}{4 B} \int_{\Omega}|\mathrm{D} z|^{4} d \gamma
\end{aligned}
$$


for any $A, B>0$. Let $\eta>0$ be such that

$$
\kappa_{1}=\frac{\eta}{q^{2} A} \quad \text { and } \quad \kappa_{2}=\frac{\eta}{2 q^{2} B} .
$$

The inequality becomes

$$
\begin{array}{r}
\frac{\eta}{c(m, p)} \mathcal{I}_{m, p}=\eta \int_{\Omega}|\mathrm{D} s|^{2} d \gamma \leq \mathcal{K}_{m, p}+\frac{A^{2} B \eta}{4} \int_{\Omega} s^{2(4-3 q)} d \gamma \\
\leq \mathcal{K}_{m, p}+\frac{\eta^{4}}{4 q^{8} \kappa_{1}^{2} \kappa_{2}} \int_{\Omega} s^{2(4-3 q)} d \gamma
\end{array}
$$

By Hölder's inequality, if $q \in(1,4 / 3)$, we obtain

$$
\int_{\Omega} s^{2(4-3 q)} d \gamma \leq\left(\int_{\Omega} s^{2(2-q)} d \gamma\right)^{\frac{4-3 q}{2-q}}
$$

since $d \gamma$ is a probability measure. Let

$$
\mathrm{K}:=\frac{1}{4 q^{8} \kappa_{1}^{2} \kappa_{2}} .
$$

The condition $q \in(1,4 / 3)$ can be rewritten as

$$
1<m<p+1
$$

Collecting all these estimates, we end up with the following estimate: for any $\eta>0$,

$$
\frac{\eta}{c(m, p)} \mathcal{I}_{m, p} \leq \mathcal{K}_{m, p}+\mathrm{K}\left[(m+p-2) \mathcal{E}_{m, p}+1\right]^{\frac{4-3 q}{2-q}} \eta^{4} .
$$

LEMMA 2.1. With the above notations, if $\Omega$ is convex, if (2.8) holds for some $\theta \in(0,1)$ and if $(m, p) \in \mathrm{E}_{\theta}$ is such that $1<m<p+1$, then

$$
\mathcal{I}_{m, p}^{\frac{4}{3}} \leq \frac{1}{3}[4 c(m, p)]^{\frac{4}{3}} \mathrm{~K}^{\frac{1}{3}}\left[(m+p-2) \mathcal{E}_{m, p}+1\right]^{\frac{4-3 q}{3(2-q)}} \mathcal{K}_{m, p} .
$$

Proof. Inequality (2.9) can be rewritten as

$$
f(\eta):=K_{1}+K_{2} \eta^{4}-K_{3} \eta \geq 0 \quad \forall \eta>0
$$

with

$$
K_{1}:=\mathcal{K}_{m, p}, \quad K_{2}:=\mathrm{K}\left[(m+p-2) \mathcal{E}_{m, p}+1\right]^{\frac{4-3 q}{2-q}} \text { and } \quad K_{3}:=\frac{\mathcal{I}_{m, p}}{c(m, p)}
$$

Optimizing on $\eta>0$, we find that

$$
0 \leq f(\bar{\eta})=K_{1}-\frac{3 K_{3}^{4 / 3}}{4^{4 / 3} K_{2}^{1 / 3}}, \quad \text { where } \quad \bar{\eta}:=\left(\frac{K_{3}}{4 K_{2}}\right)^{1 / 3}
$$

which gives the conclusion. 


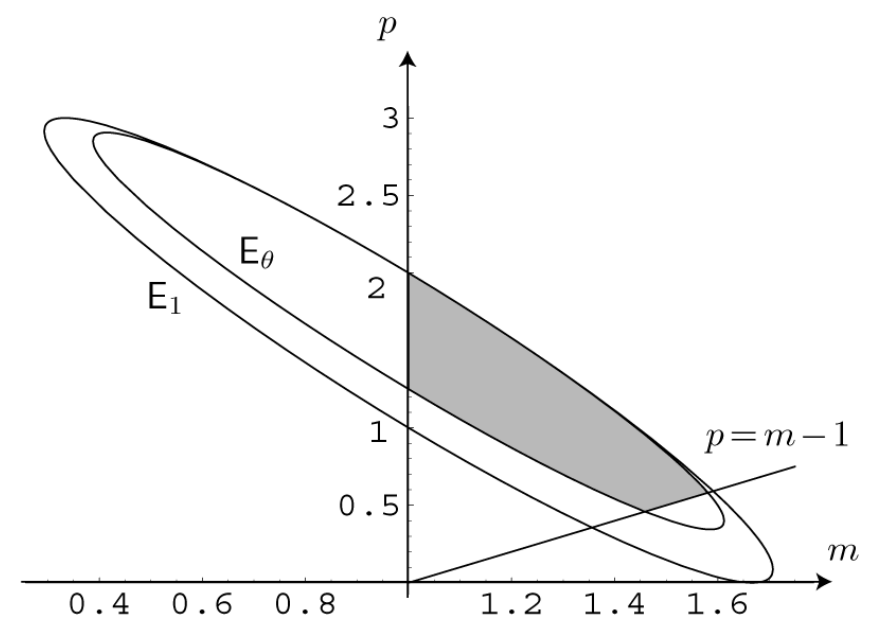

FIG. 2.1. Admissible parameters $m$ and $p$ correspond to $(m, p) \in \mathrm{E}_{\theta}, 1<m<p+1$, where the set $\mathrm{E}_{\theta}$ is defined by the condition: $\mathrm{b}^{2}-4 \mathrm{a}(\theta) \mathrm{c}<0$.

2.3. Rates of convergence and consequences. As a consequence of Lemma 2.1, we get

THEOREM 2.2. If $\Omega$ is convex, if (2.8) holds for some $\theta \in(0,1)$ and if $(m, p) \in \mathrm{E}_{\theta}$ is such that $1<m<p+1$, then there exists a positive constant $\kappa$, which depends on $\mathcal{E}_{m, p}(0)$, such that any smooth solution $u$ of (2.1) satisfies, for any $t>0$,

$$
\begin{aligned}
& \mathcal{I}_{m, p}(t) \leq \frac{\mathcal{I}_{m, p}(0)}{\left[1+\frac{\kappa}{3} \sqrt[3]{\mathcal{I}_{m, p}(0)} t\right]^{3}}, \\
& \mathcal{E}_{m, p}(t) \leq \frac{3\left[\mathcal{I}_{m, p}(0)\right]^{\frac{8}{3}}}{2 \kappa\left[1+\frac{\kappa}{3} \sqrt[3]{\mathcal{I}_{m, p}(0)} t\right]^{2}} .
\end{aligned}
$$

Proof. If $p>m-1$, then $q \in(1,4 / 3)$. Using the notations of Section 2.1 and the fact that $\mathcal{E}_{m, p}(t)$ is non-increasing, we have

$$
\frac{d}{d t} \mathcal{I}_{m, p}(t) \leq-\kappa \mathcal{I}_{m, p}^{\frac{4}{3}},
$$

with

$$
\kappa:=\frac{3}{2} m[4 \mathrm{~K} c(m, p)]^{-1 / 3}\left[(m+p-2) \mathcal{E}_{m, p}(0)+1\right]^{-\frac{4-3 q}{3(2-q)}} .
$$

An integration from 0 to $t$ gives the first estimate, and another integration from $t$ to $\infty$ gives the bound for $\mathcal{E}_{m, p}(t)$.

Constants in the above computations are explicit, but not easy to handle. However, we can observe that $\kappa$ can be written as

$$
\kappa=\kappa_{0}\left[(m+p-2) \mathcal{E}_{m, p}(0)+1\right]^{-\frac{4-3 q}{3(2-q)}},
$$


where $\kappa_{0}$ depends only on $m, p, \theta$ and $\lambda_{1}(m, \theta)$. Using the estimate for $\mathcal{E}_{m, p}$ at $t=0$ in Theorem 2.2, we obtain the following new functional inequality.

Corollary 2.3. Under the assumptions of Theorem 2.2, there exists a positive function $F$ depending only on $m, p, \theta$ and $\lambda_{1}(m, \theta)$ such that

$$
F\left(\mathcal{E}_{m, p}\right) \leq \frac{3}{2} \mathcal{I}_{m, p}^{\frac{8}{3}}
$$

where $F(s)=O(s)$ as $s \rightarrow 0_{+}$and $F(s)=O\left(s^{\frac{2}{3(2-q)}}\right)$ as $s \rightarrow \infty$.

With the above notations, we can actually write $F$ as

$$
F(s)=\kappa_{0} s[(m+p-2) s+1]^{-\frac{4-3 q}{3(2-q)}}, \quad q=\frac{p+3(m-1)}{p+2(m-1)},
$$

where the dependence of $\kappa 0$ on $m, p, \theta$ and $\lambda_{1}(m, \theta)$ can be traced explicitly in the previous computations.

\section{Conclusion}

Our method relies on Conditions (1.7) and (2.8).If (1.7) is true for some $p=p_{0} \in$ $[1,2)$, then it is also true for any $p \in\left[p_{0}, 2\right]$. Condition (1.7) for $p=p_{0}$ is equivalent to Condition (2.8) for $\theta=\theta_{0}:=\frac{2}{p_{0}}-1$, and then also holds for any $\theta \in\left(0, \theta_{0}\right)$. The domain of validity of (2.8) is represented in Fig. 2.1.

In a linear framework as well as in a non-linear setting corresponding to, respectively, (1.1) and (2.1), the Bakry-Emery method has been adapted to establish functional inequalities which extend the family of generalized Poincaré inequalities introduced by W. Beckner. As a consequence, we obtain rates of convergence for a whole family of functionals in each case. It is remarkable that the assumption on the potential can be reduced to the positivity of an eigenvalue, namely Conditions (1.7) or (2.8), that is, a condition on a linear problem, which turns out to be the same in both cases. Such a condition generalizes the Bakry-Emery criterion, in the sense that the condition is non-local. This improves on the standard criterion. Even in the non-linear case, we recover the full efficiency of the Bakry-Emery method and end up with an inequality relating an entropy functional to its associated Fisher information.

Acknowledgements. The authors thank the ANR IFO, the Procope project no. 09608ZL and University Paris Dauphine for their support.

\section{REFERENCES}

[1] L. Ambrosio, N. Gigli and G. Savaré, Gradient flows in metric spaces and in the space of probability measures, Lectures in Mathematics ETH Zürich, Birkhäuser Verlag, Basel, 2005.

[2] C. Ané, S. Blachère, D. Chafaï, P. Fougères, I. Gentil, F. Malrieu, C. Roberto and G. Scheffer, Sur les inégalités de Sobolev logarithmiques, Panoramas et Synthèses, Société Mathématique de France, Paris, 10, 2000. With a preface by Dominique Bakry and Michel Ledoux.

[3] A. Arnold, J.P. Bartier and J. Dolbeault, Interpolation between logarithmic Sobolev and Poincaré inequalities, Commun. Math. Sci., 5(4), 971-979, 2007.

[4] A. Arnold, J.A. Carrillo, L. Desvillettes, J. Dolbeault, A. Jüngel, C. Lederman, P.A. Markowich, G. Toscani and C. Villani, Entropies and equilibria of many-particle systems: an essay on recent research, Monatsh. Math., 142, 35-43, 2004.

[5] A. Arnold and J. Dolbeault, Refined convex Sobolev inequalities, J. Funct. Anal., 225, 337-351, 2005. 
[6] A. Arnold, P. Markowich, G. Toscani and A. Unterreiter, On convex Sobolev inequalities and the rate of convergence to equilibrium for Fokker-Planck type equations, Comm. Partial Differential Equations, 26, 43-100, 2001.

[7] D. Bakry, Porous media equations, personal communication, 2002.

[8] D. Bakry, Functional inequalities for Markov semigroups, Probability measures on groups: recent directions and trends, Tata Inst. Fund. Res., Mumbai, 91-147, 2006.

[9] D. Bakry and M. Émery, Diffusions hypercontractives, Séminaire de probabilités, XIX, 1983/84, Lecture Notes Math., Springer, Berlin, 1123, 177-206, 1985.

[10] J.P. Bartier and J. Dolbeault, Convex Sobolev inequalities and spectral gap, C. R. Math. Acad. Sci. Paris, 342, 307-312, 2006.

[11] W. Beckner, A generalized Poincaré inequality for Gaussian measures, Proc. Amer. Math. Soc., 105, 397-400, 1989.

[12] A. Blanchet, M. Bonforte, J. Dolbeault, G. Grillo and J.L. Vázquez, Asymptotics of the fast diffusion equation via entropy estimates, Arch. Rat. Mech. Anal., to appear.

[13] A. Blanchet, M. Bonforte, J. Dolbeault, G. Grillo and J.L. Vázquez, Hardy-Poincaré inequalities and applications to nonlinear diffusions, Comptes Rendus Mathématique, 344, 431436, 2007.

[14] A. Blanchet, J. Dolbeault and M. Kowalczyk, Stochastic Stokes' drift, homogenized functional inequalities, and large time behavior of Brownian ratchets, preprint, 2008.

[15] E.A. Carlen and M. Loss, Extremals of functionals with competing symmetries, J. Funct. Anal., 88, 437-456, 1990.

[16] J.A. Carrillo, J. Dolbeault, I. Gentil and A. Jüngel, Entropy-energy inequalities and improved convergence rates for nonlinear parabolic equations, Discrete Contin. Dyn. Syst. Ser. B, 6, 1027-1050, 2006 (electronic).

[17] J.A. Carrillo, A. Jüngel, P.A. Markowich, G. Toscani and A. Unterreiter, Entropy dissipation methods for degenerate parabolic problems and generalized Sobolev inequalities, Monatsh. Math., 133, 1-82, 2001.

[18] J.A. Carrillo, C. Lederman, P.A. Markowich and G. Toscani, Poincaré inequalities for linearizations of very fast diffusion equations, Nonlinearity, 15, 565-580, 2002.

[19] J.A. Carrillo and G. Toscani, Asymptotic $L^{1}$-decay of solutions of the porous medium equation to self-similarity, Indiana Univ. Math. J., 49, 113-142, 2000.

[20] J.A. Carrillo and J.L. Vázquez, Fine asymptotics for fast diffusion equations, Comm. Partial Differential Equations, 28, 1023-1056, 2003.

[21] D. Chafaï, Entropies, convexity, and functional inequalities: on $\Phi$-entropies and $\Phi$-Sobolev inequalities, J. Math. Kyoto Univ., 44, 325-363, 2004.

[22] P. Daskalopoulos and N. Sesum, On the extinction profile of solutions to fast-diffusion, preprint, 2006.

[23] M. Del Pino and J. Dolbeault, Best constants for Gagliardo-Nirenberg inequalities and applications to nonlinear diffusions, J. Math. Pures Appl., 81(9), 847-875, 2002.

[24] J. Demange, Porous media equation and Sobolev inequalities under negative curvature, Bull. Sci. Math., 129, 804-830, 2005.

[25] J. Denzler and R.J. McCann, Phase transitions and symmetry breaking in singular diffusion, Proc. Natl. Acad. Sci. USA, 100, 6922-6925, 2003 (electronic).

[26] J. Dolbeault, I. Gentil, A. Guillin and F.Y. Wang, $L^{q}$-functional inequalities and weighted porous media equations, Potential Anal., 28(1), 35-59, 2008.

[27] J. Dolbeault, I. Gentil and A. Jüngel, A logarithmic fourth-order parabolic equation and related logarithmic Sobolev inequalities, Commun. Math. Sci., 4, 275-290, 2006.

[28] J. Dolbeault, B. Nazaret and G. Savaré, A new class of transport distances between measures, Calc. Var \& PDE, to appear.

[29] U. Gianazza, G. Savaré and G. Toscani, The Wasserstein gradient flow of the Fisher information and the quantum drift-diffusion equation, tech. rep., Pubbl. IMATI-CNR Pavia, 2006.

[30] P. Grisvard, Elliptic problems in nonsmooth domains, Monographs and Studies in Mathematics, Pitman (Advanced Publishing Program), Boston, MA, 24, 1985.

[31] L. Gross, Logarithmic Sobolev inequalities, Amer. J. Math., 97, 1061-1083, 1975.

[32] R. Holley and D. Stroock, Logarithmic Sobolev inequalities and stochastic Ising models, J. Statist. Phys., 46, 1159-1194, 1987.

[33] R. Jordan, D. Kinderlehrer and F. Otto, The variational formulation of the Fokker-Planck equation, SIAM J. Math. Anal., 29, 1-17, 1998.

[34] A. Jüngel and D. Matthes, An algorithmic construction of entropies in higher-order nonlinear PDEs, Nonlinearity, 19, 633-659, 2006.

[35] R. Latała and K. Oleszkiewicz, Between Sobolev and Poincaré, Geometric aspects of functional 
analysis, Lecture Notes in Math., Springer, Berlin, 1745, 147-168, 2000.

[36] R.J. McCann and D. Slepčev, Second-order asymptotics for the fast-diffusion equation, Int. Math. Res. Not., Art. ID, 24947, 22, 2006.

[37] W.I. Newman, A Lyapunov functional for the evolution of solutions to the porous medium equation to self-similarity. I, J. Math. Phys., 25, 3120-3123, 1984.

[38] F. Otto, The geometry of dissipative evolution equations: the porous medium equation, Comm. Partial Differential Equations, 26, 101-174, 2001.

[39] F. Otto and M. Westdickenberg, Eulerian calculus for the contraction in the Wasserstein distance, SIAM J. Math. Anal., 37, 1227-1255, 2005 (electronic).

[40] J. Ralston, A Lyapunov functional for the evolution of solutions to the porous medium equation to self-similarity. II, J. Math. Phys., 25, 3124-3127, 1984.

[41] J.L. Vázquez, The Porous Medium Equation, Oxford Mathematical Monographs, The Clarendon Press Oxford University Press, Oxford, 2007. 